\title{
GI (GIGANTEA) Genes from Chia (Salvia hispanica): Molecular Characterization, Flowering-Related Expression and Evolutionary Features
}

\author{
Bao-Jun Chen ${ }^{1,2^{\dagger}}$, Yu-Fei Xue ${ }^{1^{\dagger}}$, Cheng-Long Yuan ${ }^{1}$, Lin Zhang', Jia-Yi Jiang', Xian-Yang Li ${ }^{1}$, Xi-Yue Luo ${ }^{3}$ and \\ You-Rong Chai ${ }^{*}$ \\ ${ }^{1}$ Chongqing Key Laboratory of Crop Quality Improvement; Chongqing Rapeseed Engineering Research Center; Engineering \\ Research Center of South Upland Agriculture of Ministry of Education; Academy of Agricultural Sciences, Southwest \\ University; College of Agronomy and Biotechnology, Southwest University, Tiansheng Road 2\#, Beibei, Chongqing, 400715, \\ China \\ ${ }^{2}$ State Key Laboratory of Cotton Biology, Institute of Cotton Research, Chinese Academy of Agricultural Sciences, Anyang, \\ 455000, China \\ ${ }^{3}$ High School Affiliated to Southwest University, Dujia Steet 43\#, Beibei, Chongqing, 400700, China \\ *For correspondence: chaiyourong@163.com \\ ${ }^{\dagger}$ Contributed equally to this work and are co-first authors \\ Received 02 March 2020; Accepted 10 June 2020; Published 10 January 2021
}

\begin{abstract}
Chia (Salvia hispanica), originated in Mexico, has outstanding nutritional and health-promoting values, but it is the only ancient American Indian staple crop failed in introduction to the Old Continents. After the rediscovery and revival of chia as a new crop in recent years, the short-day (SD) habit is a crucial limitation for its worldwide cultivation. The circadian oscillator GIGNATEA (GI) is an important transcription factor regulating flowering time through photoperiod-pathway. In this study, we cloned the full-length cDNAs of two GI genes from chia, and analyzed the molecular characteristics of the genes and the encoded proteins. Alternative transcription initiation sites, alternative poly A tailing sites, and 5'-UTR intron retention exist in some of their mRNAs. The origin of GI gene accompanied with the transition from aquatic to terrestrial habits during plant evolution. GI duplication events occurred at order, family and genus levels in angiosperms. ShGI-1 and ShGI-2 were similar to each other in organ specificity with peak expression in small buds. In mature leaf, ShGI-2 is dominant over ShGI-1 in terms of expression level with highest expression in the afternoon, but on the Autumnal Equinox day ShGI-1 is dominant over ShGI-2 with peaks at noon and in the evening. KT, BR, GA 3 and IAA upregulated the expression of ShGI-1 and ShGI-2 in long-days (LDs) and inhibited their expression in SDs, with $\mathrm{GA}_{3}$ being the most effective phytohormone. Under most abiotic stresses, ShGI expression fluctuated and returned to near-basal levels. ShGI expression was upregulated by low temperature. SA sharply upregulated ShGI expression after $24 \mathrm{~h}$ of treatment. This is the first report of GI genes from the order Lamiales, which will promote the dissection of flowering mechanism of chia and other Lamiales plants, enrich the evolution and expression characteristics of plant GIs, and promote the study on interaction between photoperiod and hormone pathways in flowering time control. (C) 2021 Friends Science Publishers
\end{abstract}

Keywords: Abiotic stresses; Chia (Salvia hispanica); Evolution; GIGNATEA (GI); Photoperiod; Phytohormones

\section{Introduction}

Since the rediscovery and revival of the ancient and sacred oil crop chia (Salvia hispanica) in recent years, it has become more and more attractive due to its high content of polyunsaturated fatty acids (PUFAs) especially $\alpha$-linolenic acid (ALA) (Sreedhar et al. 2015). Chia, an oil crop containing the highest level of ALA among the known crops, grows in deserts below 4,000 feet in Mexico and Southwest Americas, and was cultured by ancient Astek and Mayas as one of the important staple crops (Ayerza and
Coates 2005). It is also one of the most valuable crops in the Lamales order. Chia was the sacred crop of Aztecs, but the attempts have failed in introducing chia to the world since the discovery of the New World by Christopher Columbus, because it has strict short-day habit, high sensitivity to changes in photoperiods and weak tolerance to cold (Jamboonsri et al. 2012). Because of photoperiod sensitivity, the feasible geographic belts for cultivating traditional chia germplasms for grain production is restricted to $22^{\circ} 55^{\prime} \mathrm{N}-25^{\circ} 05^{\prime} \mathrm{S}$ (Hildebrand et al. 2013), and at higher latitudes the probability of the crop reaching maturity is low

To cite this paper: Chen BJ, YF Xue, CL Yuan, L Zhang, JY Jiang, XY Li, XY Luo, YR Chai (2021). GI (GIGANTEA) Genes from Chia (Salvia hispanica): Molecular Characterization, Flowering-related Expression and Evolutionary Features. Intl J Agric Biol 25:501-512 
(Ayerza and Coates 2005). Nowadays chia is commercially cultivated in several low-latitude agricultural regions in the world, mainly in Bolivia, Paraguay, Argentina, Mexico, Australia, Central America, Peru, Ecuador and Colombia, and the total acreage in 2014 was 370,000 hectares (Sosa 2016; Orona-Tamayo et al. 2017). In China, we tested chia cultivation at a $30^{\circ} \mathrm{N}$ site in winter-warm Sichuan Basin, it flowered in October, and less than one-half of the seed could reach full maturation even if we harvested it in late December (Win et al. 2018). Analyzing its flowering regulation mechanism is the basic prerequisite for creating precocious varieties and extending its cultivation to middleand high-latitude agricultural regions.

The floral induction is mainly regulated by five pathways, including photoperiod, autonomic, gibberellin, vernalization and aging pathways (Borner et al. 2000; Yuan et al. 2016; Ozturk 2017), in which the photoperiod pathway in monocots or dicots is the most conserved flowering response pathway (Yanovsky and Kay 2003). The length of day and night is perceived by photoreceptors, and the endogenous biological clock synchronizes with the environment. It is reported that GIGANTEA $(G I)$ is one of the important genes involved in normal life activities in plants. It encodes a nucleoprotein that participates in many molecular regulatory responses, such as control of circadian rhythms, transcriptional regulation of flowering, tolerance to stresses, etc. Numerous studies suggest that GI is one of the key factors controlling the plants circadian rhythm and flowering time and positively regulates the expression of downstream genes such as CO, FT and SOC1 (Mizoguchi et al. 2005; Jung et al. 2007; Duan et al. 2019; Chen et al. 2020).

With the in-depth study of $G I$, it is clear that $G I$ gene and protein sequences are quite conservative among plants. However, the structure and function of GI have not yet been fully understood (Dalchau et al. 2011; Kim et al. 2012). GI regulates gibberellin signaling through stabilization of the DELLA proteins in Arabidopsis (Nohales and Kay 2019). GI recruits the UBP12 and UBP13 deubiquitylases to regulate accumulation of the ZTL photoreceptor complex (Lee et al. 2019). HOS15 associates with a histone deacetylase complex to inhibit transcription of the GImediated photoperiodic flowering pathway in Arabidopsis (Park et al. 2019). Most modulation levels of light and temperature signaling by GI regulate the output and pace of the circadian clock (Nohales et al. 2019; Ronald et al. 2020; Park et al. 2020). Circadian process will establish the daily phasing of the behavioral, developmental, and the proper coordination of physiology and metabolism; AtGI is a cochaperone and promotes maturation of F-box protein ZEITLUPE, which is a crucial regulator of the circadian clock (Cha et al. 2017). In addition to controlling plant flowering time and circadian rhythms, GI also has numerous functions such as stress tolerance. Suárez-López et al. (2001) firstly found it as a flowering regulatory factor in Arabidopsis to activate $F T$ by regulating the transcription factor gene $C O$ in long days (LDs), allowing the plant to grow from vegetative stage to reproductive stage. At present, there are many studies about AtGI gene, which is known to play a role in drought tolerance, circadian clock control, miRNA processing, chlorophyll accumulation, light signal transmission, cold resistance, salt tolerance and herbicide resistance, besides regulating flowering time (Cao et al. 2005; Mishra and Panigrahi 2015; Cha et al. 2019). Mutation of $g i$ in Arabidopsis caused flowering delaying and increased tolerance to $\mathrm{H}_{2} \mathrm{O}_{2}$-induced oxidative stress (Fowler et al. 1999; Thiruvengadam et al. 2015), whereas overexpression of AtGl caused early flowering (Mizoguchi et al. 2005). AtGI interacted with FLAVIN-BINDING, KELCH REPEAT, AND F-BOX1 (FKF1) proteins to form the complex AtGI-AtFKF1, which promotes flowering advancement by degrading $\mathrm{CO}$ inhibitors. However, it is strange that overexpression of $O s G I$ in rice resulted in postponement of flowering time in LDs or short days (SDs), increased the expression of $H d l$, and down-regulated the expression of $H d 3 a$, which indicates that the regulatory effect of $C O$ on $F T$ in rice is the opposite of that in Arabidopsis (Hayama et al. 2003). In addition, another study of short-day plant Pharbitis nil found that overexpression of PnGI delayed flowering and PnFT1 was down-regulated (Higuchi et al. 2011). Bendix et al. (2013) studied the function of $G I(G I 1)$ in maize and found that the mutant gil promoted pre-flowering in LDs but did not show significant difference with wild-type in SDs, suggesting that wild-type GII participates in a pathway that suppresses flowering in LDs. The expression of $G I$ in Brassica oleracea was the highest at the 8 to $12 \mathrm{~h}$ of the light period and lowest at dawn under LD conditions, and downregulation of $G I$ expression in transgenic $B$. rapa enhanced salt tolerance (Thiruvengadam et al. 2015; Kim et al. 2016b). Li et al. (2013) isolated three GI genes (GmGII, GmGI2 and GmGI3) from soybean (Glycine max), GmGII had two alternative splices ( $G m G I 1 \alpha$ and $G m G I 1 \beta)$, and all GmGIs interacted with FKF1/FKF2 proteins to promote flowering. In summary, $G I$ is one of the key genes controlling flowering time, but there are significant differences in function and mechanism among different photoperiod-types of plants. GI genes were also cloned and characterized from many other crops such as longan (Dimocarpus longan), sweet potato (Dioscorea esculenta), chrysanthemum (Dendranthema morifolium), soybean and rapeseed (Brassica napus) (Li et al. 2013; Xie et al. 2015; Huang et al. 2017; Tang et al. 2017).

Chia is a revived crop with worldwide potential importance, but there are a few reports on chia about its flowering regulation mechanism. Our team is engaged in molecular dissection of the fatty acid and flowering traits of chia and the key enzyme loci FAD2 and FAD3 of ALA biosynthesis pathway as well as the flowering-related regulatory loci $C R Y$ and $S O C 1$ from chia have been reported in our previous studies (Xue et al. 2017, 2018; Chen et al. $2019,2020)$. In this study we cloned two GI genes (ShGII 
and ShGI2) from chia, analyzed their gene and protein structural features, and investigated their expression features as related to organ-specificity, diurnal dynamics, seasonal transition dynamics and responsiveness to phytohormones and abiotic stresses. Furthermore, our phylogenetic analysis also revealed some new features of plant GI evolution.

\section{Materials and Methods}

\section{Plant materials, treatment and nucleic acid extraction}

For cloning and expression study of $G I$ genes, chia plants were grown in Hechuan Farm, Southwest University, sown on May 24, 2016. On August 21-22, September 5-6, September 20-21 and October 5-6, adult leaves were sampled at 2:58, 5:58, 9:28, 12:58, 16:28, 19:58 and 23:28 of the day. They are used for gene cloning and to detect diurnal styles of gene expression. Root (Ro), stem (St), small leaves (SL), big leaves (BL), small buds (SB, about 5 days old), medium buds (MB, about 10 days old), big buds (BB, about 15 days old), flowers (Fl), early seeds (ES, about 10 days old), medium seeds (MS, about 20 days old) and late seeds (LS, about 30 days old) were sampled for detecting the organ-specificity of the cloned genes.

There were treatments with growth-stimulating phytohormones. The methods used to cultivate the seedlings of chia in the artificial climate chambers followed the reference of Xue et al. (2017). The 6-leaf stage seedlings were moved to the plant growth chambers for treatments with two styles of photoperiods. The LD treatment was 16 h-day and $8 \mathrm{~h}$-night, and the SD treatment was $12 \mathrm{~h}$-day and 12 h-night, with constant temperature of $30^{\circ} \mathrm{C}$ and relative humidity of $56 \%$. Each photoperiod treatment lasted for one week. Four hormone treatments were carried out, i.e., 80 $\mu$ mol L ${ }^{-1}$ kinetin (KT), $2 \mu$ mol L ${ }^{-1}$ brassinolide (BR), 200 $\mu \mathrm{mol} \mathrm{L}{ }^{-1}$ gibberellin $\left(\mathrm{GA}_{3}\right)$ and $250 \mu \mathrm{mol} \mathrm{L}^{-1}$ indole acetic acid (IAA) (Naeem et al. 2004). Each hormone was treated for $0 \mathrm{~d}$ (control/CK, basal level), $1 \mathrm{~d}, 3 \mathrm{~d}$ and $9 \mathrm{~d}$ respectively. Adult leaves were sampled at each time point for characterization of responsiveness of cloned genes to growth-stimulating phytohormones.

There were treatments with growth-inhibiting phytohormones and abiotic stresses. Chia seedlings were cultured in the artificial climate chamber and subjected to high temperature at $38^{\circ} \mathrm{C}$, low temperature at $4^{\circ} \mathrm{C}$, mechanical wounding, $100 \mu \mathrm{mol} \mathrm{L}{ }^{-1}$ MeJA, $100 \mu \mathrm{mol} \mathrm{L}^{-1}$ ABA, 1 mmol L ${ }^{-1} \mathrm{SA}, 300 \mathrm{mmol} \mathrm{L}^{-1}$ sodium chloride $(\mathrm{NaCl})$ and $10 \%$ polyethylene glycol 6000 (PEG6000). At 0 h, 0.5 h, 3 h, 9 h, $24 \mathrm{~h}$ and 48 h time points after treatment, adult leaf samples were taken for characterization of responsiveness of cloned genes to growth-inhibiting phytohormones and abiotic stresses (Xue et al. 2017).

In nucleic acids preparation, each study had three biological replicates. Samples were all kept in liquid nitrogen for transportation and stored at $-80^{\circ} \mathrm{C}$. Total RNA was extracted using the Biospin Plant Total RNA Extraction
Kit (BioFlux), and total gDNA was extracted from adult leaves using a CTAB method (Saghai-Maroof et al. 1984). Electrophoresis and spectrophotometric detection were adopted to detect the quality and quantity of the nucleic acids.

\section{Cloning of the conserved region sequences of chia $G I$ genes}

Since chia does not have whole-genome sequencing database and little EST, TSA, GSS and other tag sequences of chia could be found in GenBank, traditional dark-box strategy should be used to clone its genes. In order to clone the conservative regions of chia GI genes, the Arabidopsis thaliana GI mRNA (NM_102124.3) was firstly retrieved from NCBI GenBank, and used as an electron probe for the in silico cloning of $G I$ sequences from the chia-relative species such as sesame (Sesamum indicum), Erythranthe guttatus, Salvia pomifera and Salvia miltiorrhiza. All GI reference mRNA, TSA, EST and gDNA tag sequences were downloaded and multiple alignments were created. At the conservative sites of GI alignments, degenerate primer combination FLGIC + RLGIC was designed (Table 1). One $\mu \mathrm{g}$ of total RNA equal-proportionally mixed from all organs was subjected to gDNA deletion and reverse-transcribed using the PrimeScript Reagent Kit with gDNA Eraser (TaKaRa Dalian, China) to obtain the first strand library of the total cDNAs as a template for conventional Taq-PCR amplification of the conservative regions of chia GI genes (Annealing at $58^{\circ} \mathrm{C}$ and extension for $2 \mathrm{~min}$ ). Conventional electrophoresis, gel recovery, recombination with pMD19-T vector and Escherichia coli $\mathrm{DH} 5 \alpha$ transformation were performed. After PCR test for positive clones, batches of clones corresponding to insert length polymorphism were sent to Shanghai Lifei Information \& Technology Company for sequencing using M13F/M13R and walking primers.

\section{5'-RACE and 3'-RACE of chia GI genes}

To obtain the sequence information of the 5'-ends (since the transcription initiation site) and 3'-ends (before the poly A tail), we performed rapid-amplification of cDNA ends (RACE) of chia GI genes. The sequencing result of conservative region colonies signified one chia GI gene, which was named as ShGI. Then 5'-RACE and 3'-RACE primers of $S h G I$ were designed (Table 1) according to the conservative sites within the conservative region sequence. One $\mu \mathrm{g}$ of total RNA from organ-mixture was used to handle RACE procedures in terms of the usual manual of the SMARTer ${ }^{\mathrm{TM}}$ RACE Amplification Kit (Clontech, USA) to obtain the first-strand total cDNA templates of the 5'RACE and 3'-RACE. Primers FShGI3-1 and FShGI3-2 were used for pairing with the universal primers LUPM and NUP (Table 1) for 3'-RACE primary and nested amplifications of $S h G I$, respectively. The PCR annealing temperature was $64^{\circ} \mathrm{C}$ and the extension time was $1 \mathrm{~min}$. 
Primers RShGI5-1 and RShGI5-2 were matched with the universal primers LUPM and NUP (Table 1) for primary and nested amplifications of 5'-RACE of $S h G I$, respectively. The PCR annealing temperature was $62^{\circ} \mathrm{C}$ and the extension time was $1 \mathrm{~min}$. Electrophoresis, gel recovery, TA cloning and sequencing were performed.

\section{Cloning of full-length sequences of chia $G I$ genes}

Based on the sequencing results of 5'-RACE and 3'-RACE colonies, cDNA ends of two chia GI genes, ShGI-1 and ShGI-2, were produced. When they were assembled with the $S h G I$ conservative region sequence, correct matching pairs between the 5'-ends and the 3'-ends can be revealed. Then we designed the primer combinations of FShGI-1 + RShGI-1 and FShGI-2 + RShGI-2 (Table 1) for PCR amplification of the full-length sequences of the two chia GI genes, using 3'-RACE template, annealed at $62^{\circ} \mathrm{C}$, and extended for $5 \mathrm{~min}$. Electrophoresis, gel recovery, TA cloning and sequencing were performed.

\section{qRT-PCR detection of expression profiles of chia $G I$ genes}

In order to reveal the organ-specificity, photoperiod induction, phytohormone responsiveness and abiotic stress responsiveness, the transcriptional expression of ShGI-1 and ShGI-2 was detected by using primer pairs FShGI-1RT + RShGI-1RT and FShGI-2RT + RShGI-2RT, respectively. The 25SrRNA gene was detected by primer pair F25SRT + R25SRT as internal control (Table 1). The stability of the reference gene 26SRNA/25SRNA in plants was reported by a literature (Singh et al. 2004). It is one of the most conserved housekeeping genes among eukaryotes in terms of both sequence and expression. Its feasibility as an internal control in perilla and chia has been proved in our previous studies (Xue et al. 2018). The contaminated genomic DNA in the total RNA was eliminated before reverse transcription according to the manual of the PrimeScript Reagent Kit with gDNA Eraser (TaKaRa Dalian, China) with prolonged DNase treatment, and the complete digestion of DNA was ascertained by 50 cycles of PCR amplification of 25SRNA gene using the treated RNA as template, which did not generate detectable product. qRT-PCR was performed on a CFX Connect $^{\mathrm{TM}}$ Real-Time PCR Detection System (Bio-Rad, U.S.A.) with thermal cycling parameters of $95^{\circ} \mathrm{C}$ for $10 \mathrm{~min}$ and 45 cycles of amplification $\left(95^{\circ} \mathrm{C}\right.$ for $10 \mathrm{sec}, 64^{\circ} \mathrm{C}$ for 20 sec and $72^{\circ} \mathrm{C}$ for $10 \mathrm{sec}$ ). When qRT-PCR was completed, the temperature was raised from $65^{\circ} \mathrm{C}$ to $95^{\circ} \mathrm{C}$, and the melting curve was detected to confirm the specificity of the amplification. Only those results without distinct dimers and nonspecific products were used for analysis. The results with dimers or nonspecific products were abandoned, and PCRs were redone with optimized annealing temperatures and other PCR conditions until acceptable results were achieved.

\section{Bioinformatics analysis involved in this study}

Sequence creation, analysis, annotation, translation, alignments, assemblage and other analysis were mainly performed on Vector NTI Advance 11.5.1 and DNAStar version 7.1.0 softwares. In silico cloning, BLAST and CDD assays were performed on NCBI (http://www.ncbi.nlm.nih.gov), and protein analyses were performed on Expasy (http://www.expasy.org), GSDS 2.0 (http://gsds.cbi.pku.edu.cn/), CBS (http://www.cbs.dtu.dk/services/), etc. Based on multialignment, gb sequence analysis and oligo analysis on Vector NTI Advance 11.51, candidate primers were manually or automatically designed corresponding to the optimum conservative or divergent sites, and were evaluated on Primer Premier 6 to choose the best ones for practical utilization. In calculating the Tm value of the PCR primer, the Nucleic Concentration in Reaction Conditions on Primer Premier 6 was set to $100 \mathrm{nM}$. On the French website (http://www.phylogeny.fr/) (Dereeper et al. 2008), "A la Carte" mode was selected for phylogenetic tree construction. Number of bootstraps was set to 1000, until the completion of tree-building.

\section{Results}

\section{Cloning of full-length cDNAs of ShGI genes}

Electrophoresis showed that a specific $3.6 \mathrm{~kb}$ band was amplified for the conservative region of the chia GI genes. Sequencing result of three positive clones produced one member gene, and its NCBI BLASTn analysis showed highest homology to plant GI genes and was named as ShGI. No significant bands were found in the primary amplifications of 5'-RACE and 3'-RACE of ShGI, with smear at the predicted size. The 5'-RACE nested PCR of ShGI generated a band of about $400 \mathrm{bp}$. After TA cloning, all the clones had insert length polymorphisms, and sequencing results of batch clones generated the 5'-ends of two chia GI genes, named as ShGI-1 and ShGI-2 respectively. The net 5'-end lengths of ShGI-1 were 423, $390,375,366,345,303$ and $301 \mathrm{bp}$, while the net $5^{\prime}$-end lengths of ShGI-2 were 408 and 384 bp. The ShGI 3'-RACE nested PCR generated a band of about $0.5 \mathrm{~kb}$. All the clones had polymorphic insert length after TA cloning. Sequencing of batch clones produced 3'-ends of two chia GI genes. The net 3'-end lengths of ShGI-1 were 519, 452 and 441 bp, while 560 and 547 bp for ShGI-2 (Poly A not included). When assembling the conservative region sequence with the cDNA ends, correct end-to-end pairs of RACE results were obtained and PCR primer pairs were designed to amplify the full-length chia GI genes. A band of about $4 \mathrm{~kb}$ identical to the expected size was obtained in both amplifications of the full-length cDNAs of ShGI-1 and ShGI-2. We used chia total gDNA as a template to amplify the full-length gDNA of the two genes, which was unsuccessful even if we 
Table 1: Primers used in cloning and qRT-PCR detection of $G I$ genes from chia

\begin{tabular}{|c|c|c|}
\hline Primers & Sequence $\left(5^{\prime} \rightarrow 3^{\prime}\right)$ & Application \\
\hline FLGIC & СТСТСТСТААТСТСТСТССАСССААА & Forward primer for chia GI conservative regions amplification \\
\hline RLGIC & CGAACTGTAGCTGGGAGGCGACA & Reverse primer for chia GI conservative regions amplification \\
\hline FShGI3-1 & GCTTGAATGGGGAGAGTCAGGA & GSP for ShGI 3'-RACE primary amplification \\
\hline FShGI3-2 & GGGGAGAGTCAGGATTAGCAGT & GSP for ShGI 3'-RACE nested amplification \\
\hline RShGI5-1 & CATGCAAGGGCCCACTGCTC & GSP for ShGI 5'-RACE primary amplification \\
\hline RShGI5-2 & CCATGCTCCGGATGGTGAAGAAC & GSP for ShGI 5'-RACE nested amplification \\
\hline LUPM & CTAATACGACTCACTATAGGGCAAGCAGTGGTATCAACGCAGAGT & Anchor primer for 5'-and 3'-RACE primary amplification \\
\hline NUP & AAGCAGTGGTATCAACGCAGAGT & Anchor primer for 5'-and 3'-RACE nested amplification \\
\hline FShGI-1 & CTAGTTAAAGATCTCTTTCTCTCTCTCTAA & ShGI-1 full-length forward primer \\
\hline RShGI-1 & CATAGAATAATACTACAATTAATATAAATATATTATACATAC & ShGI-1 full length reverse primer \\
\hline FShGI-2 & АТТСТСТСТСССАТТТСТСТСТСТАА & ShGI-2 full-length forward primer \\
\hline RShGI-2 & GAGAGAATGAGTTATCCAAACAATAAGAAC & ShGI-2 full length reverse primer \\
\hline F25SRT & GATTTCTGCCCAGTGCTCTGAA & 25SrRNA qRT-PCR forward primer \\
\hline R25SRT & TCTGCCAAGCCCGTTCCCTT & 25SrRNA qRT-PCR reverse primer \\
\hline FShGI-1RT & TGTCGCCTCTCAGCCACC & ShGI-1 qRT-PCR forward primer \\
\hline RShGI-1RT & GTTCACGTCCGGTAGTTTGC & ShGI-1 qRT-PCR reverse primer \\
\hline FShGI-2RT & TGTCGCCTCCCAGCCACA & ShGI-2 qRT-PCR forward primer \\
\hline RShGI-2 & GTTCACA & ShGI-2 qRT-PCR reverse primer \\
\hline
\end{tabular}

replaced reagents and optimized the amplification cycle parameters, indicating that they either have very long introns or have very complex structures.

\section{Structure and features of ShGI genes}

ShGI-1 has two versions of mRNA (GenBank Accession Numbers MH107333 and MH107334, poly A not included, Fig. S1). The longest standard mRNA of ShGI-1 is $3837 \mathrm{bp}$ with 5'-UTR of $178 \mathrm{bp}$, ORF of $3504 \mathrm{bp}$ and 3'-UTR of 155 $\mathrm{bp}$, while the longest mRNA with 5'-UTR intron retention is $4067 \mathrm{bp}$ with 5'-UTR of $408 \mathrm{bp}$, ORF of $3504 \mathrm{bp}$ and 3'UTR of $155 \mathrm{bp}$. This 5'-UTR intron has non-standard splicing left border (GG...AG). ShGI-2 has longest mRNA of 3876 bp (GenBank Accession Number MH107335, poly A not included) with 5'-UTR of $163 \mathrm{bp}$, ORF of $3504 \mathrm{bp}$ and 3'-UTR of $209 \mathrm{bp}$ (Fig. S1). The G+C contents of the 5'UTR, ORF and 3'-UTR are 38.48/42.94\%, 46.89/46.99\% and $26.45 / 35.71 \%$ in $S h G I-1 / S h G I-2$, respectively. The identity percentages between ShGI-1 and ShGI-2 are 90.2\% on mRNA level and $93.5 \%$ on ORF level. BLASTn analysis shows that ShGI-1 and ShGI-2 have high homology to sesame GIGANTEA-like LOC105178750 and LOC105158892 mRNAs, E. guttatus GIGANTEA-like LOC105959402 mRNA, etc.

\section{Characterization of deduced ShGI proteins}

The ShGI-1 and ShGI-2 proteins (Fig. S1) are 1167 and 1174 aa in length, with theoretical MWs of 127.56 and $105.84 \mathrm{kD}$, pIs of 6.35 and 6.45 , respectively, which are weakly acidic. The identity percentage between the two proteins is $95.1 \%$ and the positives percentage is $96.2 \%$. BLASTp result shows that ShGI-1 and ShGI-2 have high homology with sesame GIGANTEA-like and E. guttatus GIGANTEA-like.

SignalP 4.1 (Petersen et al. 2011) prediction indicates that ShGI-1 and ShGI-2 do not contain a signal peptide.
BaCelLo (Pierleoni et al. 2006), EpiLoc (http://epiloc.cs.queensu.ca/) and Plant-mPLoc (Chou and Shen 2010) predicted the subcellular localization of ShGI-1 and ShGI-2 to be in the nuclear. SLP-Local (Matsuda et al. 2005) predicted them to be in cytoplasm or nuclear. YLoc (Hooper et al. 2014) predicted them to be in the nucleus. TMHMM2.0 (http://www.cbs.dtu.dk/services/TMHMM/) and TOPCONS (Tsirigos et al. 2015) predicted no transmembrane structures in ShGI-1 and ShGI-2. NetPhos3.1 (http://www.cbs.dtu.dk/services/NetPhos/) predicted 117/102 potential phosphorylation sites in ShGI1/ShGI-2, including 77/68 S (serine), 28/22 T (threonine) and 12/12 Y (tyrosine) sites. Summarily, both ShGI-1 and ShGI-2 proteins are most probably located in the nucleus and might be regulated by phosphorylation.

In the secondary structure of ShGI-1/ShGI-2 predicted by SOPMA (https://npsa-prabi.ibcp.fr/cgi-bin/ secpred_sopma.pl), $\alpha$-helix, $\beta$-sheet (extended strand), $\beta$ turn and random coil account for 48.93/46.69\%, $10.45 / 10.87 \%, 6.68 / 5.69 \%$ and $33.93 / 36.75 \%$, respectively (Fig. 1). The $\alpha$-helices in their proteins are nearly evenly distributed, but their locations are somewhat different between ShGI-1 and ShGI-2.

In Arabidopsis and other plants, GI plays an important role in the photoperiodic regulation of flowering (Park et al. 2013). ShGI may be involved in the regulation of flowering in chia according to ShGI protein structure, key sites in the conserved region, and physico-chemical properties.

\section{Phylogenetic relationships of GIs in plants}

In order to explore the phylogenetic relationship of plant $G I$ genes, we selected some representative species that have complete genome sequence from different taxa of plant kingdom (green algae, ferns, gymnosperms, monocots and dicots). GI protein sequences from Chia and these species were used to construct a phylogenetic tree of plant GI proteins (Fig. 2). The phylogenetic relationships are 


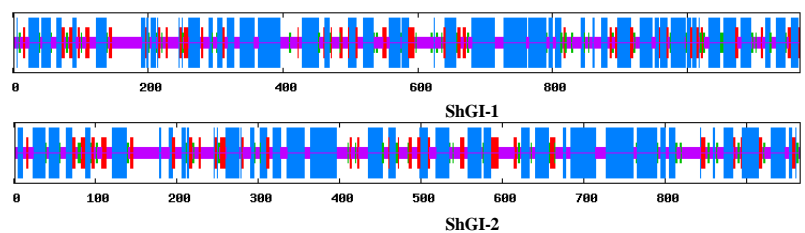

Fig. 1: Predicted secondary structures of ShGI-1 and ShGI-2

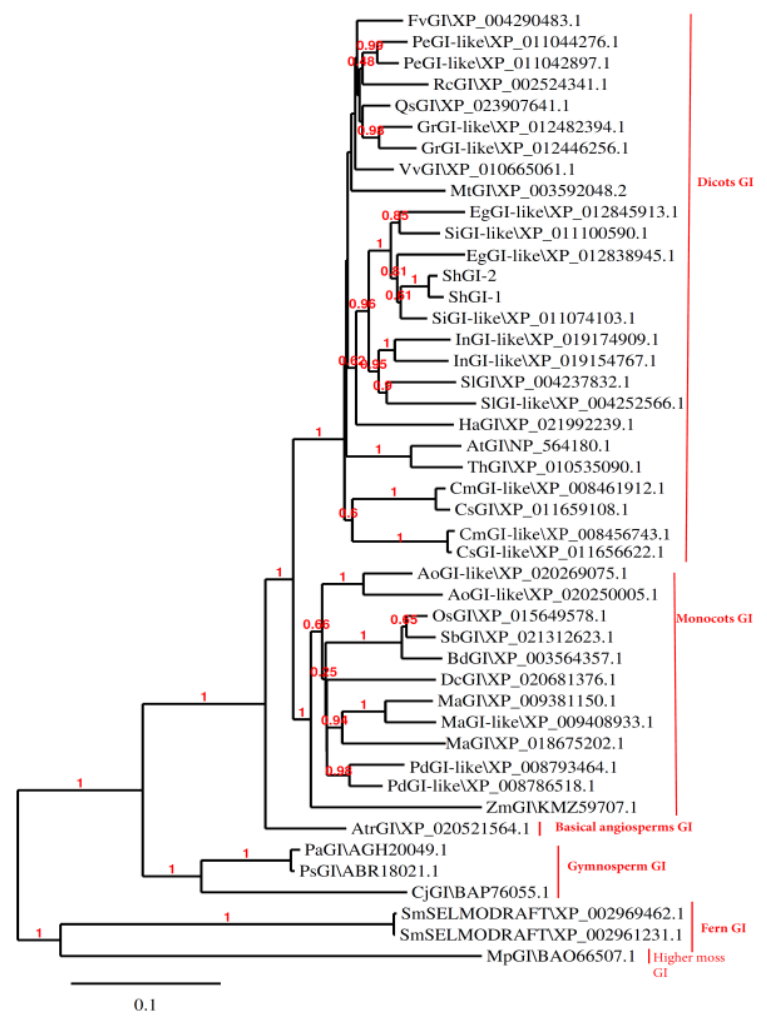

Fig. 2: Phylogenetic relationship of GI proteins from plant kingdom Ao, Asparagus officinalis; At, Arabidopsis thaliana; Atr, Amborella trichopoda; $\mathrm{Bd}$, Brachypodium distachyon; Cs, Cucumis sativus; Dc, Dendrobium catenatum; Eg, Erythranthe guttatus; Fv, Fragaria vesca; Gr Gossypium raimondii; Ha, Helianthus annuus; In, Ipomoea nil; Ma, Musa acuminate; Mt, Medicago truncatula; Pa: Picea abies; Pe, Populus euphratica; Pd, Phoenix dactylifera; Rc, Ricinus communis; Sb, Sorghum bicolor; Sh, Salvia hispanica; Si, Sesamum indicum; Sl, Solanum lycopersicum; Sm, Selaginella moellendorffii; Th, Tarenaya hassleriana; Vv, Vitis vinifera

consistent with the previous taxonomic research in the academic community, which are divided into several major groups based on the evolutionary relationships of $M$. polymorpha, Selaginella moellendorffii, gymnosperms, basal angiosperms, monocots and dicots, but some new evolutionary features of the GI genes can be observed.

There is no GI gene in aquatic lower plants such as green algae and mosses, but $G I$ genes exist in aquatic-toterrestrial transitional plant M. polymorpha, lower fern $S$. moellendorffii, gymnosperms, basal angiosperm Amborella trichopoda, monocots and dicots. This means that the origin of the GI gene was far earlier than the origin of flowering plants. It is assumed that $G I$ originated during the transition of plants from aquatic to terrestrial habits.
There is only one GI gene in Dioscorea paniculata, S. moellendorffii, gymnosperms and basal angiosperm $A$. trichopoda. Although there are two GI protein sequences cloned from $S$. moellendorffii, but they are highly similar to each other, which might be caused by the heterozygosity of the genome sequencing materials, though recent GI duplication in S. moellendorffii could not be excluded.

Basal angiosperms also have only one GI gene, and no uniform duplication of GI gene occurred in gymnosperm ancestor, angiosperm ancestor, monocot ancestor and dicot ancestor. However, GI gene duplication events occurred at angiosperm order level (e.g., Lamiales in which sesame and E. guttatus are located), family level (e.g., Malvaceae in which cotton is located) or genus and lower levels, thus many monocot and dicot species have two or more GI genes. As for genus-level GI duplication, there should be many events. For example, the well-known genome triplication in the ancestor of tribe Brassiceae would certainly lead to GI gene triplication, but this is not the focus of this study.

In Lamiales, there are two GI genes in E. guttatus and sesame; the orthologous genes from different species are clustered together, while the paralogous genes within a species are far apart, implying that an order-level GI gene duplication event occurred in Lamiales. However, the two chia GI genes cloned in this study correspond to only one $G I$ gene of E. guttatus and sesame. It is speculated that another GI gene might have been lost in chia or in Salvia genus and the remaining one experienced a recent duplication event in genus Salvia. Whether or not the orthologous gene corresponding to another GI gene of sesame and $E$. guttatus has been really deleted in genus Salvia needs to be clarified in future research.

\section{Organ-specificity of ShGI genes}

The results of qRT-PCR (Fig. 3) show that ShGI-1 is expressed in all organs but with strong organ-specificity. Its expression is very high in small buds, low in roots, stems, leaves, middle buds, big buds and seeds, and very low in stems and functional leaves. The organ-specificity of ShGI2 is similar to ShGI-1. Overall, ShGI-1 is higher than ShGI2 in expression in all organs. The latter results of this study will show that ShGI has circadian rhythm fluctuations. As each organ was detected for only one time point of the day, the organ-specificity of ShGI genes reveled here is not the complete profile of gene features.

\section{Circadian rhythms of $S h G I$ genes in response to seasonal transition}

The qRT-PCR was used to detect the circadian rhythms and the response to the seasonal change of long-short photoperiods of ShGI-1 and ShGI-2 in moderately mature functional leaves. The results showed that there were significant differences between ShGI-1 and ShGI-2 (Fig. 4). 


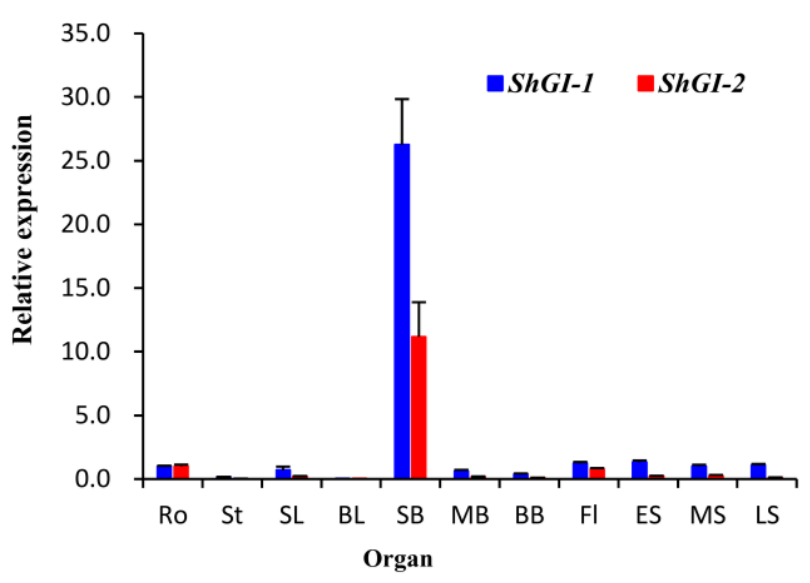

Fig. 3: Relative expression of ShGI-1 and ShGI-2 genes in different chia organs

Ro: root; St: stem; SL: small leaf; BL: big leaf; SB: small bud; MB: middle bud; BB: big bud; Fl: flower; ES: early seed; MS: middle seed; LS: late seed.

On August 21-22 (LD, sunny, $\left.28-38^{\circ} \mathrm{C}\right)$, ShGI-1 expression was low in the whole day but peaked at midnight, whereas ShGI-2 kept high level from late morning to midnight and low level from midnight to early morning. On September 56 (LD, rainy, $20-24^{\circ} \mathrm{C}$ ), both ShGI-1 and ShGI-2 were distinctly expressed from late morning to the evening with a peak in the afternoon, ShGI-2 was more distinct than ShGI-1 and from the evening to the early morning their expression was low. On September 20 and 21 (Autumnal equinox, sunny, 20-28 $8^{\circ}$ ), ShGI- 1 was distinctly expressed from the morning to the midnight with two peaks at noon and in the evening respectively, while ShGI-2 was only slightly upregulated during daytime with relatively low level, and from midnight to the early morning they both were not expressed. On October 5-6 (SDs, cloudy to overcast, 20$29^{\circ} \mathrm{C}$ ), the expression of ShGI-1 and ShGI-2 was similar to that on September 5-6, reaching a peak in the afternoon, but maintaining low levels from midnight to morning. Taken together, the two genes generally have expression peaks from late morning to midnight especially in the afternoon, the expression of $S h G I-2$ is higher than that of ShGI-1 in either LDs or SDs, while ShGI-1 is dominant over ShGI-2 on the Autumnal equinox day especially at noon and evening peaks.

\section{Effects of phytohormones on expression patterns of ShGI genes in LDs and SDs}

In this study, $\mathrm{KT}, \mathrm{BR}, \mathrm{GA}_{3}$ and IAA treatments were performed on 6-leaf stage chia seedlings in LDs and SDs, respectively. The expression changes of ShGI-1 and ShGI-2 were detected by qRT-PCR (Fig. 5). There were also differences and similarities between/among photoperiods, hormones and genes. In BR treatment, ShGI-1 and ShGI-2 were firstly dramatically upregulated in LDs, and then slightly fell back. However, ShGI-1 and ShGI-2 were dramatically down-regulated by BR in SDs, then kept at low levels. In $\mathrm{GA}_{3}$ treatment, ShGI-1 and ShGI-2 were significantly upregulated in LDs, but its effect was slower than that of BR; conversely, they were rapidly downregulated in SDs, and then stayed at low levels. In IAA treatment, ShGI-1 and ShGI-2 were gradually upregulated in LDs. However, ShGI-1 and ShGI-2 firstly were inhibited slightly by IAA in SDs, and then returned to basal levels with even a little upregulation. In KT treatment, ShGI-1 and $S h G I-2$ were significantly increased to and kept at a certain level in LDs, while in SDs they were down-regulated and restored soon. Taken together, in the chia leaf, ShGI genes are promoted to varying degrees in LDs by phytohormones $\mathrm{BR}$, KT, IAA and $\mathrm{GA}_{3}$, but are inhibited by these phytohormones to varying degrees in SDs, with $\mathrm{GA}_{3}$ being the most effective phytohormone. LD is opposite to SD in manifesting the effects of phytohormones on ShGI expression in chia leaf.

\section{Expression patterns of ShGI genes under various abiotic stresses}

According to reports, GI regulates the circadian rhythm, growth and development of plants, and responses to salt stress, and thus has important basal functions. However, its response to other abiotic stresses is rarely reported. We used 5-week old chia seedlings to perform multiple stress treatments and detected changes in the expression of ShGI-1 and ShGI-2 based on qRT-PCR (Fig. 6). The expression of ShGI-1 and ShGI-2 was similar to each other under various stresses. After cold treatment at $4^{\circ} \mathrm{C}$, expression of ShGI-I and ShGI-2 slightly fluctuated within $48 \mathrm{~h}$ with an overall trend of upregulation, and ShGI-1 was more sensitive than ShGI-2. At $38^{\circ} \mathrm{C}$ heat stress, ShGI-1 and ShGI-2 were temporarily sharply upregulated and quickly returned to basal levels. After MeJA treatment, ShGI-1 and ShGI-2 were upregulated dramatically and then slowly fell back to reach basal levels at $48 \mathrm{~h}$. After mechanical wound, ABA and $\mathrm{NaCl}$ treatments, ShGI-I and ShGI-2 were firstly downregulated, and then fluctuated with recovery or even upregulation, but the overall trends were downregulation. After PEG treatment, ShGI-1 and ShGI-2 were relatively stable in expression with a little upregulation. ShGI-1 and ShGI-2 responded to SA treatment very slowly, but after 24 $\mathrm{h}$ they were significantly upregulated, especially for ShGI-1.

\section{Discussion}

In this study, we isolated and molecularly characterized the two GI genes from chia, which is a recalcitrant short-day crop rediscovered recently. The two ShGI genes show typical structural features, and some features of plant $G I$ gene origination and evolution are revealed. According to qRT-PCR results, ShGI-1 and ShGI-2 both are dominantly expressed in small buds, and are regulated by various internal and external signals with distinct responsiveness patterns especially opposite effects between LD and SD 


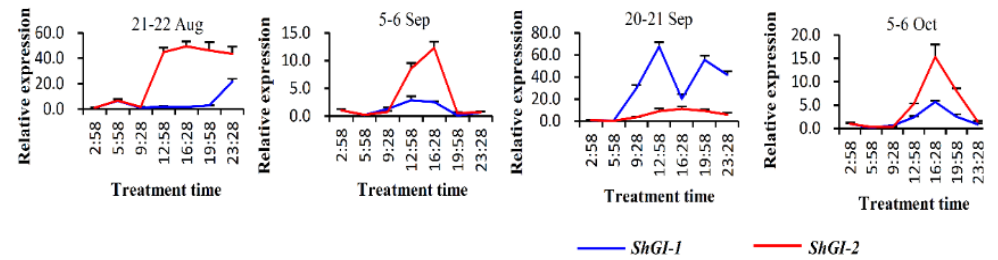

Fig. 4: Circadian rhythm of ShGI-1 and ShGI-2 expression, and response to long-short photoperiod seasonal changing


Fig. 5: Influence of important flowering hormones on the expression of ShGI-1 and ShGI-2 in long-short period
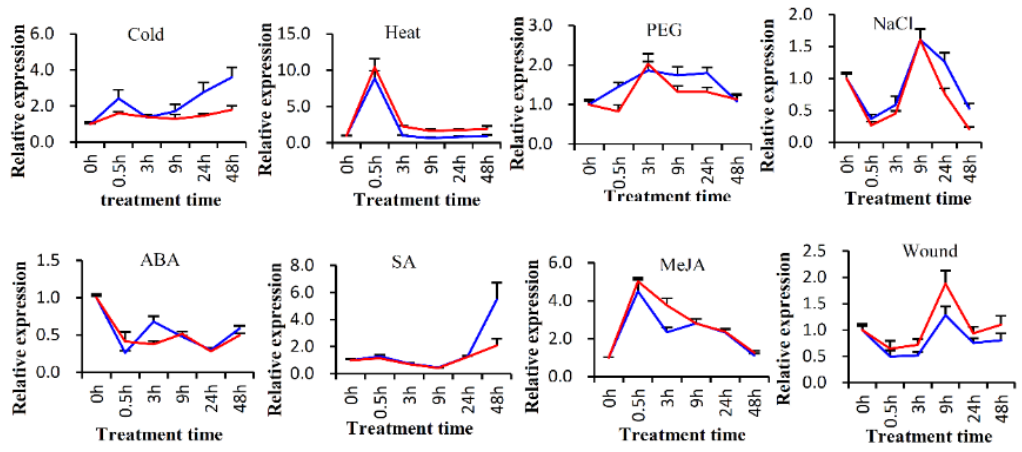

ShGI-I $\quad$ ShGI-2

Fig. 6: Influence of abiotic stresses on the expression of ShGI-1 and ShGI-2

photoperiods. Among these results, we mainly discuss the following major aspects.

\section{The effects of phytohormones on expression of ShGI depend on the photoperiod condition}

A large number of studies have shown that the phytohormones are involved in the regulation of flowering time, especially the gibberellin signaling pathway is one of the five major pathways of flowering induction. The hormonal pathway interacts with the photoperiodic and the vernalization pathway reactions (Seo et al. 2011). In tree peony of forcing culture, $\mathrm{GA}_{3}$-hormone changes promoted PSSOC1 and PSSPL9 expression, and repressed PSSVP expression, which contributed to the improvement flowering quality (Guan et al. 2019). Although GI is involved in the regulation of many physiological functions, including flowering time, the report of its response to the phytohormones is not systematic. In view of this, in this study four phytohormones were used to treat chia seedlings in LDs and SDs, respectively. ShGI-1 and ShGI-2 were similar to each other in response characteristics. KT, BR, $\mathrm{GA}_{3}$ and IAA promoted ShGI-1 and ShGI-2 in LDs, and inhibited them in SDs. $\mathrm{GA}_{3}$ has the strongest effect among the four phytohormones.

This study shows that the effects of phytohormones on GI expression depend on photoperiod, and the effect in LDs is contrary to that in SDs. This finding will promote the study on the interaction between photoperiod and hormonal pathways. At present, there lacks report on the effects of phytohormones on $G I$ expression in both LDs and SDs within a study. In this study, we systematically reveal ShGI 
expression as influenced by four phytohormones in both LDs and SDs, which provides a reference to other researchers to dive into GI regulation mechanisms.

\section{ShGI expression changes in response to seasons and various abiotic stresses}

In chia leaf, the expression of ShGI-2 was higher than that of ShGI-1 in LDs and SDs, high in the afternoon but low from midnight to the early morning, and was less influenced by rainy. However, from morning to midnight on the Autumnal equinox day, ShGI-1 expression was dominant over ShGI-2. The expression of AtGI was high in the afternoon, low in the morning and at night in Arabidopsis in LDs or SDs (Mizoguchi et al. 2005; David et al. 2006; Paltiel et al. 2006; Rubio and Deng 2007; Sawa et al. 2007; Dalchau et al. 2011; Sawa and Kay 2011; Han et al. 2013). The AtGI gene was transformed into Chinese cabbage with an expression high in the afternoon and low in the morning and at night, regardless of day length (Xie et al. 2015; Kim et al. 2016a). Both in long-day plants Annona squamosal, Medicago truncatula and Populus alba, and in short-day plants $P$. nil and Ipomoea batatas, the expression of GI was high in the afternoon and low in the morning and evening in LDs or SDs (Paltiel et al. 2006; Ke et al. 2017; Tang et al. 2017; Barros et al. 2017). The P. nil GI in the dark continues to retain the same pattern in LDs or SDs, indicating strict biological clock control (Higuchi et al. 2011). It can be seen that the circadian rhythmic characteristics of $G I$ in the plant kingdom are conserved among species and among gene members and the two chia ShGI genes have circadian rhythmic characteristics similar to other plants.

Some abiotic stresses also have an effect on the expression of ShGI-1 and ShGI-2. The response of ShGI-1 and ShGI-2 was slow after SA treatment, but they were significantly upregulated after $24 \mathrm{~h}$ especially for ShGI-1. After MeJA treatment, the expression of ShGI-1 and ShGI-2 increased dramatically and gradually returned to the basal levels. After cold treatment at $4^{\circ} \mathrm{C}, S h G I-1$ and $S h G I-2$ were slightly upregulated, and $S h G I-1$ was more sensitive. In heat treatment at $38^{\circ} \mathrm{C}$, they immediately returned to basal levels after transient upregulation. After mechanical injury, ABA and $\mathrm{NaCl}$ treatments, the expression of ShGI-1 and ShGI-2 was first downregulated and then fluctuated, with an overall trend of a little downregulation. After PEG treatment, the expression of ShGI-1 and ShGI-2 was relatively stable with a little upregulation. Overall, some adversities have a certain influence on the expression of ShGI. The expression of $G I$ was slightly upregulated when the $P$. alba plants were treated with high concentrations of $\mathrm{NaCl}$, and Arabidopsis flowering time was generally delayed after the PagGI was transformed into Arabidopsis (Ke et al. 2017). The expression of $I b G I$ in sweetpotato was down-regulated under cold treatment, but upregulated under heat treatment, and both drought and $\mathrm{NaCl}$ treatments upregulated $I b G I$
(Tang et al. 2017). Reducing the expression of GI in transgenic rapeseed enhanced plants tolerance to $\mathrm{NaCl}$ (Kim et al. 2016b). The ABA-dependent signal gene AtGI participated in escaping drought in Arabidopsis by upregulating FT and advancing flowering (Riboni et al. 2016). When sprayed with high or low concentrations of $\mathrm{NaCl}$, Arabidopsis plants with gi deletion had stronger salt tolerance, while plants with overexpression of GI had the weakest salt tolerance (Park et al. 2013). Besides regulating plant growth and flowering, $\mathrm{GA}_{3}$ can also amend antioxidant enzyme and osmotic regulation to improve salt tolerance of okra (Zhu et al. 2019). GA 3 and GI might have important mutual interactions to coordinate growth and development with stress tolerance. This study reveals the effects of eight abiotic stresses on the expression of ShGI genes in Chia, which is helpful to further study the relationship between adversity and chia flowering and other traits, and also enriches the understanding of the plants $G I$ expression characteristics.

\section{Evolutionary characteristics of plant $G I$ genes}

In this study, we selected representative species that have complete genome sequences from different taxa of plant kingdom, constructed a phylogenetic tree of GI proteins, and revealed some new features of the GI evolution in the plant kingdom. This study indicates that green algae and mosses have no $G I$, and $G I$ is present in both $M$. polymorpha and higher plants. GI originated during the transition process of plants from aquatic habit to terrestrial habit, far earlier than the origin of flowering plants. Though gymnosperms are not considered to have true flowers, recent studies indicate that many of the flowering genes are present in gymnosperms (Mao et al. 2019). Obviously, the occurrence of $G I$ was not originally to produce flowering traits, but rather to be involved in regulating development and adaptability of terrestrial plants which evolved more complicated traits than aquatic ancestor plants. The function of $G I$ is to regulate phyB signaling pathways, biological clocks, flowering time, carbohydrate metabolism, seasonality in growth and cold tolerance (Cao et al. 2005; Kim et al. 2016a, 2017; Ding et al. 2018). It is even possible to discover in the future that GI regulates more aspects of growth and adaptability of terrestrial plants. GI exists far earlier than the origin of flowering plants, and its function should be far more than regulating flowering. It is speculated that regulating flowering is only a derived function from the original function of $G I$ after its origin.

This study found that the duplication events of $G I$ genes occurred in the evolutionary process within some orders, families and genera of angiosperms. There is no common duplication of $G I$ across the plant kingdom, and thus only one $G I$ gene exists in $M$. polymorpha, $S$. moellendorffii, gymnosperms and basal angiosperm $A$. trichopoda. Some monocot or dicot species still have only one GI gene, but some other species have two or more GI 
genes. One GI duplication event occurred in the early period of Lamiales evolution, resulting in two GI genes in $E$. guttatus and sesame. However, the two ShGI genes cloned in this study were the result of a recent duplication in the genus Salvia. Whether the ortholog of another GI gene of $E$. guttatus and sesame has been lost in Chia and other Salvia species needs further cloning study to reach a conclusion. In related previous reports, generally only one $G I$ gene for each species was selected (Ke et al. 2017; Tang et al. 2017), therefore it was not possible to effectively reveal the full-set evolutionary features of plant $G I$ genes. For the first time, this study systematically reveals the evolutionary features of GIs in plant kingdom.

Studies have shown that GI regulates flowering, and activates the flowering gene $F T$ by regulating the transcription factor gene $C O$ in the flowering pathway of LDs, so that the plant enters the reproductive stage. When the longan DlGI gene was transformed into Arabidopsis, the flowering time was greatly advanced (Huang et al. 2017). When the poplar PagGI gene was transformed into Arabidopsis, it promoted the expression of $C O$ and $F T$ genes and caused early flowering ( $\mathrm{Ke}$ et al. 2017), while the absence of AtGI caused late flowering of Arabidopsis (Tang et al. 2017). Since studies have shown that GI is a positive regulator of flowering in response to photoperiods by controlling circadian rhythms, and affects plants' resistance to stresses, it is speculated that ShGI family may also participate in flowering induction and regulate other physiological functions. Similar to GIs from sweetpotato, poplar, chrysanthemum, poplar, Arabidopsis, etc. (Ke et al. 2017; Tang et al. 2017), the two chia GI genes also have strong organ-specificity with dominant expression in small buds, suggesting that the $G I$ gene regulates not only flowering time but also bud primordium differentiation.

$G I$ is a promoting factor for flowering in the long-day plant $A$. thaliana, but in the short-day plant chia it is promoted by four phytohormones in LDs and inhibited by them in SDs. How to link this rule with the mechanism of short-day activation of flowering in Chia, and the mechanism of flowering regulation of typical short-day plants, needs to be studied in depth. In addition, the responses of the chia GI family to circadian rhythms, seasonal changes and abiotic stresses also suggest that these environmental factors may affect flowering or other reproductive traits by affecting the expression of $G I$, especially that the associations of chia $G I$ genes with SA signaling and cold tolerance deserve special attention in the future.

ShGI-1 and ShGI-2 are similar to each other in most expression characteristics and in the protein structures, therefore their protein activities and basic physiological functions should also be similar, with redundancy and additive effects. However, they do have distinct differences in circadian rhythm and seasonal changes as well as a little difference in response to phytohormones and abiotic stresses, implying functional divergence in regulating flowering time.

\section{Conclusion}

GI originated during the transition from aquatic habit to terrestrial habit of plants, and GI duplications occurred only in angiosperm orders, families and genera. Fulllength cDNAs of two GI genes possibly regulating photoperiod-pathway flowering have been cloned from the revived short-day crop Chia. The 3837-bp ShGI-I mRNA and 3876-bp ShGI-2 mRNA and their encoded proteins have typical structural and molecular features. ShGI-1 and ShGI-2 both have dominant expression in small buds, and are regulated by photoperiod, phytohormones and abiotic stresses. In mature leaf, ShGI2 is dominant over ShGI-1 with highest expression in the afternoon, but on the Autumnal Equinox day ShGI-1 is dominant over $S h G I-2$ with peaks at noon and in the evening. KT, BR, $\mathrm{GA}_{3}$ and IAA upregulate $S h G I-1$ and ShGI-2 in LDs and inhibit them in SDs, with $\mathrm{GA}_{3}$ being the strongest phytohormone. Low temperature and SA upregulate ShGI expression, and other abiotic stresses also exert influences.

\section{Acknowledgements}

This study was supported by the Chongqing Research Program of Basic Research and Frontier Technology (cstc2015jcyjBX0143), National Key R\&D Program of China (2016YFD0100506), the Fundamental Research Funds for the Central Universities (No.XDJK2014D009), and the Chongqing Youth Innovative Talent Training Eaglet Project (CY170203, CY150212).

\section{Author Contributions}

Conceived and designed the experiments: Bao-Jun Chen and You-Rong Chai. Performed the experiments: Bao-Jun Chen, Yu-Fei Xue, Cheng-Long Yuan, Lin Zhang, Jia-Yi Jiang and Xi-Yue Luo. Analyzed the data: Bao-Jun Chen, Yu-Fei Xue and Xian-Yang Li. Wrote the paper: Bao-Jun Chen and You-Rong Chai.

\section{References}

Ayerza R, W Coates (2005). Chia: Rediscovering a Forgotten Crop of the Aztecs. The University of Arizona Press, Tucson, Arizona, USA

Barros PM, S Cherian, M Costa, H Sapeta, NJM Saibo, MM Oliveira (2017). The identification of almond GIGANTEA gene and its expression under cold stress, variable photoperiod, and seasonal dormancy. Biol Plantarum 61:631-640

Bendix C, JM Mendoza, DN Stanley, R Meeley, FG Harmon (2013). The circadian clock-associated gene giganteal affects maize developmental transitions. Plant Cell Environ 36:1379-1390

Borner R, G Kampmann, J Chandler, R Gleiner, E Wisman, K Apel, S Melzer (2000). A MADS domain gene involved in the transition to flowering in Arabidopsis. Plant J 24:591-599

Cao S, M Ye, S Jiang (2005). Involvement of GIGANTEA gene in the regulation of the cold stress response in Arabidopsis. Plant Cell Rep $24: 683-690$ 
Cha JY, DY Lee, I Ali, SY Jeong, B Shin, H Ji, JS Kim, MG Kim, WY Kim (2019). Arabidopsis GIGANTEA negatively regulates chloroplast biogenesis and resistance to herbicide butafenacil. Plant Cell Rep 38:793-801

Cha JY, J Kim, TS Kim, QN Zeng, L Wang, SY Lee, WY Kim, DE Somers (2017). GIGANTEA is a co-chaperone which facilitates maturation of ZEITLUPE in the Arabidopsis circadian clock. Nat Commun 8; Article 3

Chen BJ, YF Xue, XL Liao, CL Yuan, L Zhang, X Yang, ML Jiang, YX $\mathrm{Qu}$, YR Chai (2020). Cloning and expression features of flowering integrator genes ShSOC1-1 and ShSOC1-2 from Chia (Salvia hispanica). Intl J Agric Biol 23:951-962

Chen BJ, YF Xue, BT Wang, XF Shi, FR Mei, AN Win, YR Chai (2019). Cloning and expression features of cryptochrome genes, ShCRY1 and ShCRY2, from chia (Salvia hispanica). Intl J Agric Biol 22:866-876

Chou KC, HB Shen (2010). Plant-mPLoc: A top-down strategy to augment the power for predicting plant protein subcellular localization. PLoS One 5: Article e11335

Dalchau N, SJ Baek, HM Briggs, FC Robertson, AN Dodd, MJ Gardner, MA Stancombea, MJ Haydona, GB Stanc, JM Gonçalvesd, AAR Webb (2011). The circadian oscillator gene GIGANTEA mediates a long-term response of the Arabidopsis thaliana circadian clock to sucrose. Proc Natl Acad Sci USA 108:5104-5109

David KM, U Armbruster, N Tama, J Putterill (2006). Arabidopsis GIGANTEA protein is post-transcriptionally regulated by light and dark. FEBS Lett 580:1193-1197

Dereeper A, V Guignon, G Blanc, S Audic, S Buffet, F Chevenet, JF Dufayard, S Guindon, V Lefort, M Lescot, JM Claverie, O Gascuel (2008). Phylogeny.fr: Robust phylogenetic analysis for the nonspecialist. Nucl Acids Res 36:465-469

Ding J, H Böhlenius, MG Rühl, P Chen, S Sane, JA Zambrano, B Zheng, ME Eriksson, O Nilsson (2018). GIGANTEA-like genes control seasonal growth cessation in Populus. New Phytol 218:1491-1503

Duan XQ, JD Liang, P Wang (2019). Overexpression of SOC1-like gene promotes flowering and decreases seed set in Brachypodium. Intl $J$ Agric Biol 22:234-242

Fowler S, K Lee, H Onouchi, A Samach, K Richardson, B Morris, G Coupland, J Putterill (1999). GIGANTEA: A circadian clockcontrolled gene that regulates photoperiodic flowering in Arabidopsis and encodes a protein with several possible membranespanning domains. EMBO J 18:4679-4688

Guan YR, JQ Xue, YQ Xue, RW Yang, SL Wang, XX Zhang (2019). Effect of exogenous $\mathrm{GA}_{3}$ on flowering quality, endogenous hormones, and hormone- and flowering-associated gene expression in forcing-cultured tree peony (Paeonia suffruticosa). J Integr Agric 18:1295-1311

Han Y, X Zhang, Y Wang, F Ming (2013). The suppression of WRKY44 by GIGANTEA-miR172 pathway is involved in drought response of Arabidopsis thaliana. PLoS One 8; Article e73541

Hayama R, S Yokoi, S Tamaki, M Yano, K Shimamoto (2003). Adaptation of photoperiodic control pathways produces short-day flowering in rice. Nature 422:719-722

Higuchi Y, K Sage-Ono, R Sasaki, N Ohtsuki, A Hoshino, S lida, H Kamada, M Ono (2011). Constitutive expression of the GIGANTEA ortholog affects circadian rhythms and suppresses one-shot induction of flowering in Pharbitis nil, a typical short-day plant. Plant Cell Physiol 52:638-650

Hildebrand D, W Jamboonsri, T Phillips (2013). Early flowering mutant chia and uses thereof. United States Patent US8586831B2

Hooper CM, SK Tanz, IR Castleden, MA Vacher, ID Small, AH Millar (2014). SUBAcon: A consensus algorithm for unifying the subcellular localization data of the Arabidopsis proteome. Bioinformatics 30:3356-3364

Huang F, Z Fu, L Zeng, M Morley-Bunker (2017). Isolation and characterization of $G I$ and $F K F 1$ homologous genes in the subtropical fruit tree Dimocarpus longan. Mol Breed 37; Article 90

Jamboonsri W, TD Phillips, RL Geneve, JP Cahill, DF Hildebrand (2012). Extending the range of an ancient crop, Salvia hispanica L.- a new $\omega-3$ source. Genet Resour Crop Evol 59:171-178
Jung JH, YH Seo, PJ Seo, JL Reyes, J Yun, NH Chua, CM Park (2007). The GIGANTEA-regulated microRNA172 mediates photoperiodic flowering independent of CONSTANS in Arabidopsis. Plant Cell 19:2736-2748

Ke Q, HS Kim, Z Wang, CY Ji, JC Jeong, HS Lee, YI Choi, BC Xu, XP Deng, DJ Yun, SS Kwak (2017). Down-regulation of GIGANTEAlike genes increases plant growth and salt stress tolerance in poplar. Plant Biotechnol J 15:331-343

Kim JA, HS Kim, SH Choi, JY Jang, MJ Jeong, SI Lee (2017). The importance of the circadian clock in regulating plant metabolism. Intl J Mol Sci 18:2680-2690

Kim HM, JH Lee, AY Kim, SH Park, SH Ma, S Lee, YH Joung (2016a). Heterologous expression of an RNA-binding protein affects flowering time as well as other developmental processes in Solanaceae. Mol Breed 36:71-85

Kim JA, H Jung, JK Hong, V Hermand, CR McClung, YH Lee, JY Kim, SI Lee, MJ Jeong, JS Kim, DJ Yun, WY Kim (2016b). Reduction of GIGANTEA expression in transgenic Brassica rapa enhances salt tolerance. Plant Cell Rep 35:1943-1954

Kim Y, M Yeom, H Kim, J Lim, HJ Koo, D Hwang, D Somers, HG Nam (2012). GIGANTEA and EARLY FLOWERING 4 in Arabidopsis exhibit differential phase-specific genetic influences over a diurnal cycle. Mol Plant 5:678-687

Lee CM, MW Li, A Feke, W Liu, AM Saffer, JM Gendron (2019). GIGANTEA recruits the UBP12 and UBP13 deubiquitylases to regulate accumulation of the ZTL photoreceptor complex. Nat Commun 10; Article 3750

Li F, X Zhang, R Hu, F Wu, J Ma, Y Meng, Y Fu (2013). Identification and molecular characterization of $F K F 1$ and $G I$ homologous genes in soybean. PLoS One 8; Article 79036

Mao D, J Ye, F Xu (2019). Advances of the flowering genes of gymnosperms. Not Bot Hortic Agrobot 47:1-9

Matsuda S, JP Vert, H Saigo, N Ueda, H Toh, T Akutsu (2005). A novel representation of protein sequences for prediction of subcellular location using support vector machines. Protein Sci 14:2804-2813

Mishra P, KC Panigrahi (2015). GIGANTEA-an emerging story. Front Plant Sci 6; Article 8

Mizoguchi T, L Wright, S Fujiwara, F Cremer, K Lee, H Onouchi, A Mouradov, S Fowler, H Kamada, J Putterill, G Coupland (2005). Distinct roles of GIGANTEA in promoting flowering and regulating circadian rhythms in Arabidopsis. Plant Cell 17:2255-2270

Naeem M, IRAM Bhatti, RH Ahmad, MY Ashraf (2004). Effect of some growth hormones $\left(\mathrm{GA}_{3}\right.$, IAA and kinetin) on themorphology and early or delayed initiation of bud of lentil (Lens culinaris Medik). Pak J Bot 36:801-809

Nohales MA, SA Kay (2019). GIGANTEA gates gibberellin signaling through stabilization of the DELLA proteins in Arabidopsis. Proc Natl Acad Sci USA 116:21893-21899

Nohales MA, W Liu, T Duffy, K Nozue, M Sawa, JL Pruneda-Paz, JN Maloof, SE Jacobsen, SA Kay (2019). Multi-level modulation of light signaling by GIGANTEA regulates both the output and pace of the circadian clock. Dev Cell 49:840-851

Orona-Tamayo D, ME Valverde, O Paredes-López (2017). Chia-the new golden seed for the $21^{\text {st }}$ century: Nutraceutical properties and technological uses. In: Sustainable Protein Sources, pp:265-281. Nadathur S (Ed.). Academic Press, Amsterdam, The Netherlands

Ozturk N (2017). Phylogenetic and functional classification of the photolyase/cryptochrome family. Photochem Photobiol 93:104-111

Paltiel J, R Amin, A Gover, N Ori, A Samach (2006). Novel roles for GIGANTEA revealed under environmental conditions that modify its expression in Arabidopsis and Medicago truncatula. Planta 224:1255-1268

Park HJ, WY Kim, DJ Yun (2013). A role for GIGANTEA: Keeping the balance between flowering and salinity stress tolerance. Plant Signal Behav 8; Article e24820

Park HJ, D Baek, JY Cha, X Liao, SH Kang, CR McClung, SY Lee, DJ Yun, WY Kim (2019). HOS15 interacts with the histone deacetylase HDA9 and the evening complex to epigenetically regulate the floral activator GIGANTEA. Plant Cell 31:37-51 
Park YJ, JY Kim, JH Lee, BD Lee, NC Paek, CM Park (2020). GIGANTEA shapes the photoperiodic rhythms of thermomorphogenic growth in Arabidopsis. Mol Plant 13:459-470

Petersen TN, S Brunak, GV Heijne, H Nielsen (2011). SignalP 4.0: Discriminating signal peptides from transmembrane regions. Nat Meth 8:785-786

Pierleoni A, PL Martelli, P Fariselli, R Casadio (2006). BaCelLo: A balanced subcellular localization predictor. Bioinformatics 22:408-416

Riboni M, RA Test, M Galbiati, C Tonelli, L Conti (2016). ABA-dependent control of GIGANTEA signaling enables drought escape via upregulation of FLOWERING LOCUS T in Arabidopsis thaliana. $J$ Exp Bot 67:6309-6322

Ronald J, K McCarthy, SJ Davis (2020). GIGANTEA integrates photoperiodic and temperature signals to time when growth occurs. Mol Plant 13:357-359

Rubio V, XW Deng (2007). Plant science: Standing on the shoulders of GIGANTEA. Science 318:206-207

Saghai-Maroof MA, KM Soliman, RA Jorgensen, RW Allard (1984). Ribosomal DNA spacer-length polymorphisms in barley: Mendelian inheritance, chromosomal location, and population dynamics. Proc Natl Acad Sci USA 81:8014-8018

Sawa M, SA Kay (2011). GIGANTEA directly activates Flowering Locus $T$ in Arabidopsis thaliana. Proc Natl Acad Sci USA 108:11698-11703

Sawa M, DA Nusinow, SA Kay, T Imaizumi (2007). FKF1 and GIGANTEA complex formation is required for day-length measurement in Arabidopsis. Science 318:261-265

Seo E, J Yu, KH Ryu, MM Lee, I Lee (2011). WEREWOLF, a regulator of root hair pattern formation, controls flowering time through the regulation of FT mRNA stability. Plant Physiol 156:1867-1877

Singh K, J Raizada, P Bhardwaj, S Ghawana, A Rani, H Singh, K Kaul, S Kumar (2004). 26S rRNA-based internal control gene primer pair for reverse transcription-polymerase chain reaction-based quantitative expression studies in diverse plant species. Anal Biochem 335:330-333

Sreedhar RV, P Kumari, SD Rupwate, R Rajasekharan, M Srinivasan (2015). Exploring triacylglycerol biosynthetic pathway in developing seeds of Chia (Salvia hispanica L.): A transcriptomic approach. PLoS One 10: Article e0123580

Sosa A (2016). Chia crop (Salvia hispanica L.): Its history and importance as a source of polyunsaturated fatty acids omega-3 around the world: A review. J Crop Res Fert 1:1-9
Suárez-López P, K Wheatley, F Robson, H Onouchi, F Valverde, G Coupland (2001). CONSTANS mediates between the circadian clock and the control of flowering in Arabidopsis. Nature 410:1116-1120

Tang W, H Yan, ZX Su, SC Park, YJ Liu, YG Zhang, X Wang, M Kou, DF Ma, SS Kwak, Q Li (2017). Cloning and characterization of a novel GIGANTEA gene in sweet potato. Plant Physiol Biochem 116:27-35

Thiruvengadam M, CF Shih, CH Yang (2015). Expression of an antisense Brassica oleracea GIGANTEA (BoGI) gene in transgenic broccoli causes delayed flowering, leaf senescence, and post-harvest yellowing retardation. Plant Mol Biol Rep 33:1499-1509

Tsirigos KD, C Peters, N Shu, L Käll, A Elofsson (2015). The TOPCONS web server for consensus prediction of membrane protein topology and signal peptides. Nucl Acids Res 43:401-407

Win AN, YF Xue, BJ Chen, FF Liao, F Chen, NW Yin, FR Mei, BT Wang, XF Shi, YM He, YR Chai (2018). Chia (Salvia hispanica) experiment at a $30^{\circ} \mathrm{N}$ site in Sichuan Basin, China. Cienc Rur 48:113

Xie Q, P Lou, V Hermand, R Aman, HJ Park, DJ Yun, WY Kim, MJ Salmela, BE Ewers, C Weinig, SL Khan, DLP Schaible, CR McClung (2015). Allelic polymorphism of GIGANTEA is responsible for naturally occurring variation in circadian period in Brassica rapa. Proc Natl Acad Sci USA 112:3829-3834

Xue YF, BJ Chen, AN Win, C Fu, JP Lian, X Liu, R Wang, XC Zhang, YR Chai (2018). Omega-3 fatty acid desaturase gene family from two $\omega$ 3 sources, Salvia hispanica and Perilla frutescens: Cloning, characterization and expression. PLoS One 13:1-25

Xue YF, NW Yin, BJ Chen, FF Liao, AN Win, JY Jiang, R Wang, XY Jin, N Lin, YR Chai (2017). Molecular cloning and expression analysis of two FAD2 genes from chia (Salvia hispanica). Acta Physiol Plantarum 39; Article 95

Yanovsky MJ, SA Kay (2003). Living by the calendar: How plants know when to flower. Nat Rev Mol Cell Biol 4:265-276

Yuan S, ZW Zhang, C Zheng, ZY Zhao, Y Wang, LY Feng, GQ Niu, CQ Wang, JH Wang, H Feng, F Xu, F Bao, Y Hu, Y Cao, LG Ma, HY Wang, DD Kong, W Xiao, HH Lin, YK He (2016). Arabidopsis cryptochrome 1 functions in nitrogen regulation of flowering. Proc Natl Acad Sci USA 113:7661-7666

Zhu G, J Yin, X Guo, X Chen, W Zhi, J Liu, Y Wang, H Lu, X Jiao, G Zhou (2019). Gibberellic acid amended antioxidant enzyme and osmotic regulation to improve salt tolerance of okra at early growth stage. Intl J Agric Biol 22:270-276 